\title{
A New Na+-Dependent RNA-Cleaving DNAzyme with over 1000-fold Rate Acceleration by Ethanol
}

\author{
Wenhu Zhou, ${ }^{[a, b]}$ Runjhun Saran, ${ }^{[b]}$ Qingyun Chen, ${ }^{[b]}$ Jinsong Ding ${ }^{[a]}$ and Juewen Liu ${ }^{* a, b]}$ \\ Dedication ((optional))
}

\begin{abstract}
Enzymes working in organic solvents are important for analytical chemistry, catalysis, and mechanistic studies. While a few protein enzymes are highly active in organic solvents, little is known regarding nucleic acid based enzymes. Herein, we report the first RNA-cleaving DNAzyme named EtNa that works optimally in concentrated organic solvents containing only monovalent $\mathrm{Na}^{+}$. The EtNa DNAzyme has a rate of $2.0 \mathrm{~h}^{-1}$ in $54 \%$ ethanol (with $120 \mathrm{mM}$ $\mathrm{NaCl}$ and no divalent metal ions), and a $K_{\mathrm{d}}$ of $21 \mathrm{mM} \mathrm{Na}^{+}$. It is still active even in $72 \%$ ethanol and also in DMSO. With $4 \mathrm{mM} \mathrm{Na}^{+}$, the rate in $54 \%$ ethanol is $>1000$-fold than that in water. Using EtNa for measuring the ethanol content in alcoholic drinks is also demonstrated. Taken together, this DNAzyme has three unique features: divalent metal independent activity, $\mathrm{Na}^{+}$selectivity among monovalent metals, and acceleration by organic solvents.
\end{abstract}

Keywords: DNAzymes • organic solvents • sodium • RNA cleavage $\cdot$ biosensors

DNAzymes refer to DNA molecules with catalytic activity. ${ }^{[1-3]}$ In the past two decades, DNAzymes played a critical role in biosensor development, ${ }^{[4-7]}$ and nanotechnology. ${ }^{[8-10]}$ This is largely attributable to their excellent stability, cost-effectiveness, high catalytic efficiency, and programmability. So far, most DNAzyme-related work was carried out in aqueous solutions, while the effect of organic solvents was rarely explored. Studying DNAzymes in organic solvents can expand their analytical applications for detecting target molecules in a broader range of sample matrix.

DNA is soluble and can maintain its base pairing in many organic solvents up to a certain solvent concentration. ${ }^{[11-13]}$ For example, with increasing ethanol concentration, DNA duplex is initially destabilized as indicated by a decreased DNA melting temperature $\left(T_{m}\right)^{\left[{ }^{[14]}\right.}$ At a critical ethanol concentration (depending on the type and concentration of cations present), DNA starts to aggregate. For such aggregated DNA (still in the B-form), its $T_{\mathrm{m}}$ is no longer affected by salt concentration. When the ethanol concentration reaches up to $70-80 \%$, a B-to-A transition takes place due to extensive DNA dehydration.

Most of the previous work focused on duplex stability and DNA hybridization kinetics in organic solvents, ${ }^{[15-18]}$ while research on DNA catalysis is rarely explored. ${ }^{[19-22]}$ Recently, a RNA-ligating DNAzyme was selected in $10 \%$ methanol and tested in organic solvents. ${ }^{[23]}$ We are interested in RNA-cleaving DNAzymes since they are more versatile in applications. In this work, we report a new RNA-cleaving DNAzyme showing the best activity in $54 \%$ ethanol. It is also active in a few other organic solvents including DMSO (dimethyl sulfoxide). At the same time, this DNAzyme does not require any divalent metal ions for catalysis and it works optimally with $\mathrm{Na}^{+}$. The use of this DNAzyme for measuring the alcohol content is also demonstrated.

Our discovery of this new DNAzyme was accidental. Most RNAcleaving DNAzymes require divalent metal ions for catalysis. ${ }^{[21,24,25]}$ We recently expanded the metal cofactor and isolated a few trivalent lanthanide-dependent DNAzymes. ${ }^{[26-29]}$ In our attempt to further explore the chemistry of RNA-cleaving DNAzymes, we wondered whether metal complexes such as hemin can also assist this RNA cleavage reaction. Hemin is an iron containing porphyrin and a common biological cofactor. Many G-quadruplex structures bind to hemin, ${ }^{[30]}$ and these binding complexes have the peroxidase activity. ${ }^{[31,32]}$ However, hemin has not yet been tested for other types of DNAzyme reactions.

The selection was carried out using our previously established protocols with a DNA library containing 50 random nucleotides. ${ }^{[26-28]}$ See Table S1 for the DNA sequences and modifications used in this work. The intended cleavage site involved a single adenine ribonucleotide $(\mathrm{rA})$ since RNA is more susceptible to cleavage. ${ }^{[33,34]}$ To induce cleavage, $50 \mu \mathrm{M}$ hemin was added to the library (Figure $1 \mathrm{~A}$ step 1). The cleaved sequences were isolated by gel electrophoresis (step 2) and amplified by two PCR steps (steps 3,4 ). In the second PCR (step 4), the P4 primer contains a polymer spacer to stop PCR extension. Therefore, two strands of unequal lengths were produced. The shorter strand was harvested using gel electrophoresis (step 5), and then precipitated in isopropanol (step 6) to seed the next round of selection. The selection conditions are detailed in Table S2.

[a] W. Zhou, Prof. Dr. J. Ding, Prof. Dr. Liu

School of Pharmaceutical Sciences, Central South University,

Changsha, Hunan 410013, China

[b] W. Zhou, R. Saran, Q. Chen, Prof. Dr. J. Liu

Department of Chemistry and Waterloo Institute for Nanotechnology

University of Waterloo

200 University Avenue West, Waterloo, Ontario, Canada, N2L 3G1

Fax: (+)1 519-746-0435

E-mail: liujw@uwaterloo.ca 

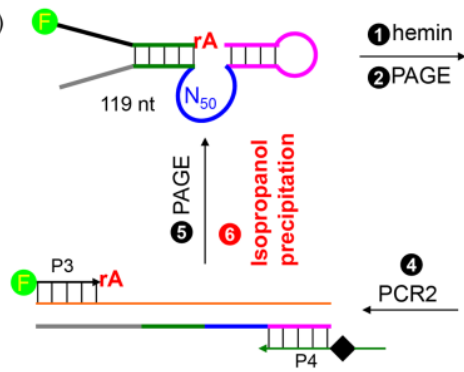

C)
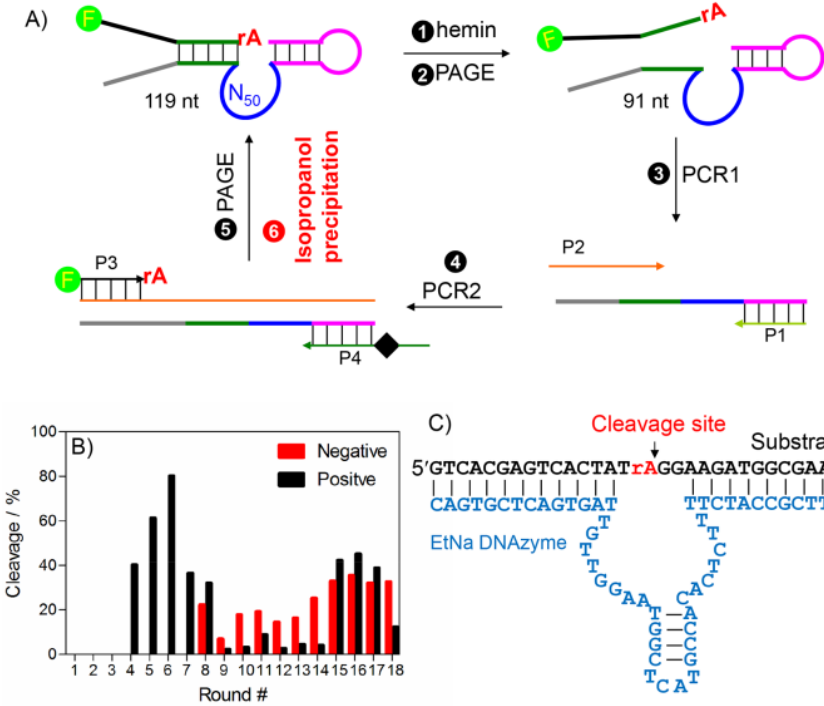

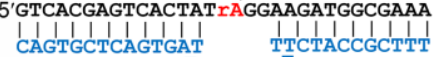
EtNa DNAZyme $G^{\text {T }}$ EtNa DNAzyme

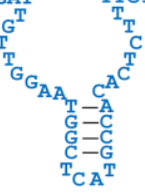

Figure 1. (A) A scheme of the in vitro selection process. PAGE stands for polyacrylamide gel electrophoresis. The key step responsible for this particular work is step 6: isopropanol precipitation. Cleavage occurred at this step instead of at the intended step 1. The cleaved sequences are amplified by two rounds of PCR to seed the next round of selection. (B) The cleavage percentage of the library at each round of selection. The red bars indicate negative selections, where cleavage was intended to be induced by the selection buffer alone (no hemin). (C) The secondary structure of the EtNa DNAzyme. The cleavage site in the substrate is marked by the arrowhead.

No cleavage occurred in the first three rounds (Figure 1B). At round $4, \sim 40 \%$ cleavage was observed and the yield increased to $80 \%$ at round 6 . However, we found that the cleavage was independent of hemin at round 7 (i.e. similar cleavage was observed using the selection buffer alone). To remove such non-specific sequences, we then performed ten rounds of negative selection (red bars, Figure 1B), where the cleaved library in the absence of hemin was discarded and the remaining library was extracted and incubated with hemin for the positive selection. At the end of round 18, however, the buffer alone still yielded $\sim 40 \%$ cleavage, indicating failure of the negative selection strategy.

While the hemin-dependent activity was not achieved, the library appeared to be quite active with the buffer alone and we decided to investigate the origin of cleavage. The round 18 library was cloned and sequenced. The obtained 34 sequences aligned remarkably well and 30 of them were essentially identical (see Table S3 for the alignment). The folding of a representative sequence is shown in Figure S1, which was easily engineered to a trans-cleaving DNAzyme, named EtNa (Figure 1C). This is a typical DNAzyme structure with two base paired regions for substrate binding. The catalytic core contains a hairpin and two unpaired bulges.

However, no cleavage of the substrate was observed in the selection buffer ( $50 \mathrm{mM}$ MES, $\mathrm{pH} 6.0,25 \mathrm{mM} \mathrm{NaCl}$ ). To solve this puzzle, we thought carefully about our selection process. The isopropanol precipitation step (Figure 1B, step 6) involved a final of $75 \%$ isopropanol with $200 \mathrm{mM} \mathrm{NaOAc}$ and $80 \mu \mathrm{M} \mathrm{MgCl}$ for overnight incubation. We suspected that cleavage might have occurred during this step. In other words, the organic solvent might be important for catalysis.
To test this hypothesis, the EtNa DNAzyme complex was added to increasing concentrations of ethanol (all with $120 \mathrm{mM} \mathrm{NaCl}$ ). Indeed, the fraction of cleavage increased significantly with increasing ethanol concentration up to $54 \%$ (Figure $2 \mathrm{~A}$ ). Beyond that, the activity started to drop. To test generality, the same reaction was carried out in methanol and isopropanol. Indeed, the DNAzyme was active in all these alcoholic solvents and the activity peaked at $\sim 54 \%$ solvent concentration (see Figure $2 \mathrm{C}$ for quantification). Noticeably, the enzyme remained quite active even in $72 \%$ solvents. We next measured the cleavage kinetics in $54 \%$ of the alcohols (Figure 2D). The rates are between 0.019 and $0.033 \mathrm{~min}^{-1}$ in $\mathrm{pH} 6.0 \mathrm{MES}$ buffer with $120 \mathrm{mM} \mathrm{NaCl}$. It appears that the type of alcohol is not critical, but an alcoholic environment is important.

A)
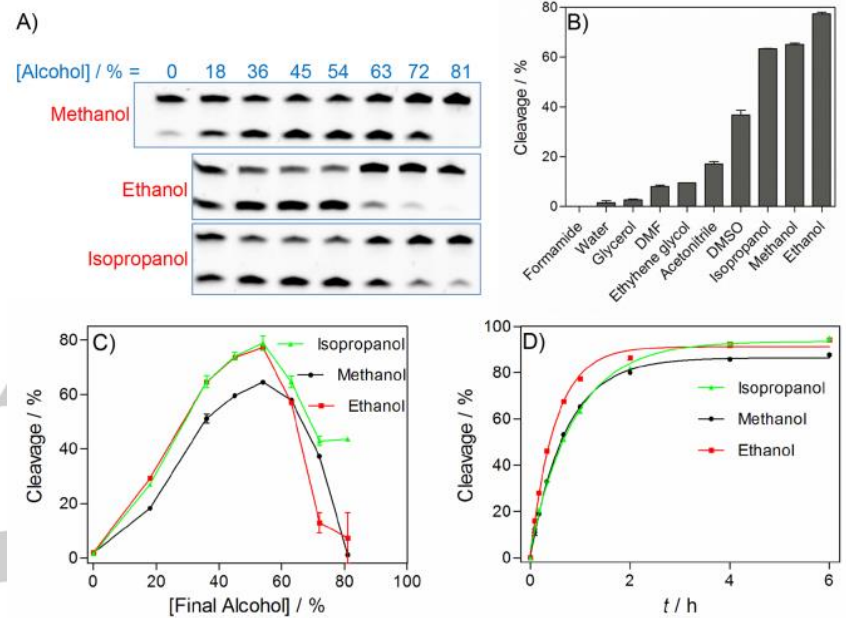

Figure 2. (A) Gel images showing cleavage of the EtNa DNAzyme as a function of solvent concentration in methanol, ethanol and isopropanol after $1 \mathrm{~h}$ incubation. (B) Fraction of substrate cleavage after $1 \mathrm{~h}$ in various solvents (54\%). (C) Quantification of the gel data in (A). (D) Cleavage kinetics of the EtNa DNAzyme with $54 \%$ methanol, ethanol or isopropanol. The reaction buffer contained $50 \mathrm{mM} \mathrm{MES}(\mathrm{pH}$ 6.0) and $120 \mathrm{mM} \mathrm{NaCl}$ for all the reactions.

This study explains the cleavage in our selection (i.e. the library was cleaved during the isopropanol precipitation step). The property of duplex DNA in alcoholic solvents has been well studied; the B-form duplex can be maintained up to $70 \%$ ethanol. ${ }^{[14]}$ After that, DNA is dehydrated and converts to the A-form. This transition might be responsible for the drastically decreased DNAzyme activity beyond $72 \%$ ethanol. In addition, DNA precipitation at high ethanol concentrations (e.g. $>75 \%$ ) might also contribute to the loss of activity.

We then tested a few other water miscible solvents at $54 \%(\mathrm{v} / \mathrm{v})$ concentration (Figure 2B). Among these, DMSO also showed moderate activity, while cleavage in the other solvents was less than $20 \%$ after $1 \mathrm{~h}$. Therefore, this enzyme works the best in the alcohols. This might be attributable to our selection conditions. Since the EtNa DNAzyme appears to be a new and interesting enzyme, we studied it further. Ethanol was chosen in the subsequent studies since it is a more common solvent than isopropanol.

Most DNAzymes require divalent or trivalent metal ions for activity. During our selection, the isopropanol precipitation step was carried out with a high concentration of $\mathrm{Na}^{+}(200 \mathrm{mM})$ and a trace amount of $\mathrm{Mg}^{2+}(80 \mu \mathrm{M})$. All the above assays were carried out in $\mathrm{NaCl}$ only without divalent metal ions. To fully understand its metal ion requirement, we studied monovalent ions first. Gel images of a few 
monovalent salt dependent reactions are shown in Figure 3A. Among these, $\mathrm{Na}^{+}$produced the most cleavage. The cleavage results are quantified in Figure 3B. Higher concentrations of $\mathrm{Na}^{+}$produced more cleavage, while all the other salts suppressed cleavage at concentrations beyond $20 \mathrm{~mm}$. The second highest cleavage was observed with $\mathrm{Rb}^{+}$followed by $\mathrm{K}^{+}, \mathrm{Li}^{+}, \mathrm{NH}_{4}^{+}$, and $\mathrm{Cs}^{+}$. The specificity for $\mathrm{Na}^{+}$might also be attributed to the selection condition. Recently, another $\mathrm{Na}^{+}$-specific DNAzyme was reported by the Lu group. ${ }^{[35]}$

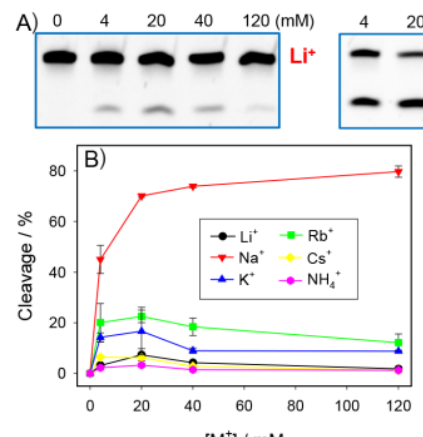

$\left[\mathrm{M}^{+}\right] / \mathrm{mM}$
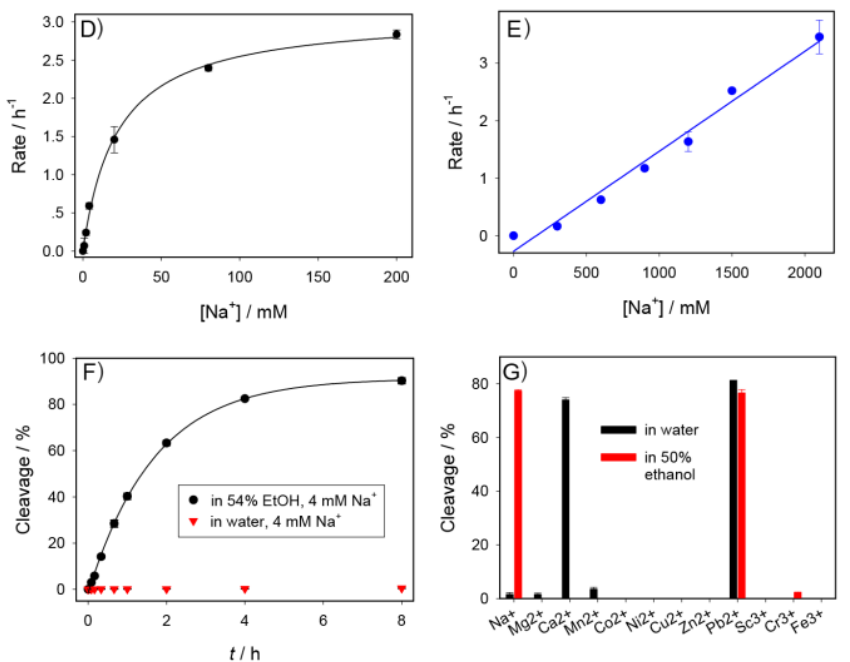

Figure 3. (A) Gel images of the EtNa DNAzyme cleavage with various concentrations of $\mathrm{Li}^{+}, \mathrm{Na}^{+}$and $\mathrm{Cs}^{+}$after $1 \mathrm{~h}$ reaction in $54 \%$ ethanol. (B) Fraction of substrate cleavage as a function of monovalent salt concentration after $1 \mathrm{~h}$ in 54\% ethanol. (C) Cleavage kinetics in the presence of various concentrations of $\mathrm{Na}^{+}$in $54 \%$ ethanol. (D) Cleavage rate of $\mathrm{EtNa}$ in $54 \%$ ethanol as a function of $\mathrm{Na}^{+}$ concentration. (E) Cleavage rate of EtNa in water (no ethanol) as a function of $\mathrm{Na}^{+}$concentration. (F) Kinetics of substrate cleavage with 4 $\mathrm{mM} \mathrm{NaCl}$ in $54 \%$ ethanol or in aqueous solution. (G) Selectivity EtNa with various divalent and trivalent metal ions in water or in $50 \%$ ethanol. The cleavage was measured in the presence of $2 \mathrm{mM}$ divalent metal ions, $0.2 \mathrm{mM}$ trivalent metal ions, or $100 \mathrm{mM} \mathrm{Na}{ }^{+}$. The DNAzyme was initially annealed in $50 \mathrm{mM}$ Tris buffer containing $120 \mathrm{mM} \mathrm{Li}{ }^{+}, \mathrm{pH}$ 7.5 to avoid background cleavage.

While most nucleic acid enzymes require divalent or trivalent metal ions for activity, enzymes independent of such metal ions are also known in a few cases. ${ }^{[35-40]}$ However, the effect of solvent was not explored in these systems. We believe this EtNa DNAzyme has provided a scaffold to study the interaction between metal ions and DNA in organic solvents. It has also added another example of monovalent-dependent DNAzymes.
We then measured $\mathrm{Na}^{+}$-concentration-dependent cleavage kinetics in 54\% ethanol (Figure 3C). No cleavage was observed with just $0.8 \mathrm{mM} \mathrm{Na}^{+}$. This might be due to the lack of DNA hybridization in such low salt condition. Even $2 \mathrm{mM} \mathrm{Na}{ }^{+}$produced over $70 \%$ cleavage in 8 h. Significantly faster cleavage was observed with higher $\mathrm{NaCl}$ concentrations, reaching a rate of $2.8 \mathrm{~h}^{-1}$ with $200 \mathrm{mM} \mathrm{NaCl}$. We then plotted the cleavage rate as a function of $\mathrm{NaCl}$ concentration (Figure $3 D)$, and an apparent dissociation constant $\left(K_{\mathrm{d}}\right)$ of $21 \mathrm{mM} \mathrm{NaCl}$ was obtained. For comparison, the rate was linearly increased with increasing $\mathrm{Na}^{+}$concentration in water, and no saturation was observed even with $2.1 \mathrm{M} \mathrm{Na}^{+}$, suggesting a quite non-specific binding or low affinity (Figure 3E). EtNa achieved a rate slope of $0.0019 \mathrm{~h}$ 1. $\mathrm{mM}^{-1} \mathrm{Na}^{+}$in water, and $0.14 \mathrm{~h}^{-1} \cdot \mathrm{mM}^{-1} \mathrm{Na}^{+}$in $54 \%$ ethanol, which is $\sim 74$-fold tighter.

Next we tested a low salt concentration of only $4 \mathrm{mM} \mathrm{NaCl}$ (Figure $3 F)$. At this condition, almost no cleavage was observed in water, and the effect of ethanol is very pronounced. The estimated rate enhancement by ethanol is over 1000 -fold. It is possible that the local $\mathrm{Na}^{+}$concentration around the DNAzyme in ethanol is higher (due to the smaller dielectric constant of ethanol and thus stronger electrostatic attraction), and this may promote the enzyme activity. An interesting example on the hairpin ribozyme was reported by Seyhan and Burke, ${ }^{[41]}$ who noted that a partially hydrated ribozyme film can still retain some cleavage activity with only monovalent $\mathrm{Na}^{+}$, although the rate was very slow ( 2 day $^{-1}$ under optimized conditions). Dehydrate was achieved by ethanol precipitation and vacuum centrifugation, which were the same operations carried out by us in our in vitro selection experiments. The hairpin ribozyme was not optimized for $\mathrm{Na}^{+}$under the ethanol precipitation conditions, but our EtNa DNAzyme was isolated under this condition, explaining its higher activity.

All our data so far indicate that the EtNa DNAzyme works in monovalent (in particular $\mathrm{Na}^{+}$) ions alone. To rule out the presence of any divalent salts, the reaction was also carried out in $20 \mathrm{mM}$ EDTA which chelates any possible divalent metals, and similar cleavage was also observed (Figure S2).

We further measured the effect of divalent and trivalent metal ions both in aqueous and ethanol containing solution (Figure $3 \mathrm{G}$ ). In aqueous solution, only $2 \mathrm{mM} \mathrm{Pb}^{2+}$ and $\mathrm{Ca}^{2+}$ showed substantial cleavage, while in ethanol, only $\mathrm{Pb}^{2+}$ can cleave. Overall, $\mathrm{Na}^{+}$is quite specific for this DNAzyme, both in aqueous solution and in ethanol.

While the main goal of this work is to report this new DNAzyme and its initial characterization, we also explored a preliminary application here. Development of simple yet effective methods for detection of ethanol content is important since ethanol plays indispensable roles in clinical, industrial, biochemical and food applications. For example, as one of the most important parameters in fermentation processes, alcohol content indicates the final quality of alcoholic beverages. The current standard methods, such as HPLC, ${ }^{[42]}$ and gas chromatography $(\mathrm{GC})^{[43]}$ are able to detect alcohol content with high sensitivity and selectivity. However, these methods often suffer from disadvantages such as sophisticated instrumentations, time-consuming processes and complicated operation steps. Therefore, researchers have explored alternative approaches using chemical and biosensors, and a few alcohol sensors based on small molecules have been reported. ${ }^{[44-46]}$ 
Since EtNa shows an interesting relationship between its activity and alcohol content, we reason that EtNa should be a useful probe for alcohol content detection. To test this, a quantitative assay was carried out. To ensure consistent enzyme activity, all the samples were mixed with a $\mathrm{Na}^{+}$-containing MES buffer prior to adding EtNa. With increasing ethanol concentration, the substrate cleavage was increased, and a linear response was observed with a dynamic range of $0-60 \%$ (Figure $4 \mathrm{~A}$ ). Note the ethanol concentration in the $x$-axis of this plot refers to the initial concentration, and the final concentration in the reaction mixture was diluted by half. The lower concentration range cleavage is shown in the inset. From this, we calculated the detection limit to be $3 \%$ ethanol based on three times of background variation divided by the slope.
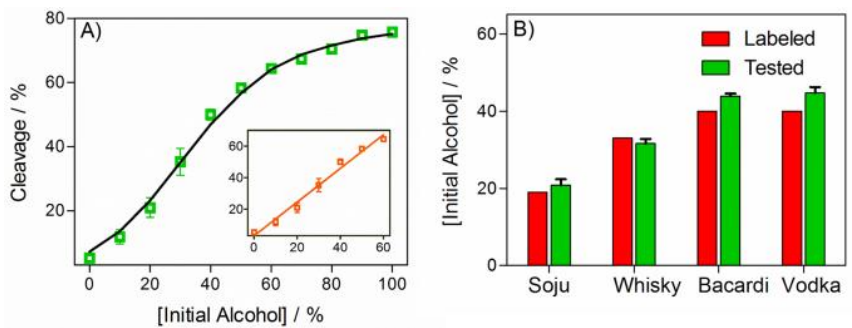

Figure 4. (A) A calibration curve for alcohol content measurement using EtNa. Inset: the linear response at low alcohol contents. (B) Detection of alcohol content in four commercial alcoholic beverages. The red bars are from the labels and green bars are from our DNAzyme measurement.

Using this calibration curve, a few commercial alcoholic beverages containing different concentrations of labeled ethanol were further analysed using the same protocol. For all the tested samples, the alcohol content measured by our method is similar to that of labeled alcoholic content (within 5\% difference), indicating EtNa is useful in measuring alcohol content in quite complex sample matrix (Figure 4B).

\section{Conclusions}

In summary, we performed an in vitro selection experiment with hemin as the intended cofactor. A new RNA-cleaving DNAzyme named EtNa was unintentionally obtained, which is active in alcoholic solvents requiring only $\mathrm{Na}^{+}$. Compared to its activity in water, this DNAzyme can be significantly accelerated by concentrated organic solvents. This is particularly true at low $\mathrm{Na}^{+}$concentrations. In addition, this DNAzyme shows interesting metal specificity. Among all the monovalent metals tested, $\mathrm{Na}^{+}$provides the highest activity. None of the higher valent metal ions produce significant activity except for $\mathrm{Ca}^{2+}$ and $\mathrm{Pb}^{2+}$. The application of EtNa in measuring alcohol content was demonstrated, and it worked even in commercial wines. Taken together, this new DNAzyme has brought up three interesting observations: monovalent metal activity, sodium ion selectivity, and accelerated rates by concentrated organic solvents. It has expanded the scope of functional nucleic acids research and applications to organic solvents.

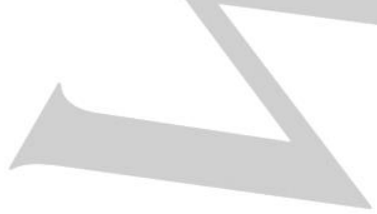

\section{Acknowledgements}

This work is supported by the University of Waterloo, the Natural Sciences and Engineering Research Council of Canada (NSERC), Foundation for Shenghua Scholar of Central South University and the National Natural Science Foundation of China (Grant No. 21301195). W. Zhou is supported by a Fellowship from the China Scholarship Council (CSC, Grant No. 201406370116).

[1] G. F. Joyce, Ann. Rev. Biochem. 2004, 73, 791.

[2] M. Famulok, J. S. Hartig, G. Mayer, Chem. Rev. 2007, $107,3715$.

[3] D. S. Wilson, J. W. Szostak, Annu. Rev. Biochem. 1999, 68, 611.

[4] J. Liu, Z. Cao, Y. Lu, Chem. Rev. 2009, 109, 1948

[5] X.-B. Zhang, R.-M. Kong, Y. Lu, Annu. Rev. Anal. Chem. 2011, 4, 105.

[6] K. Schlosser, Y. F. Li, Chem. Biol. 2009, 16, 311.

[7] S. K. Silverman, Acc. Chem. Res. 2009, 42, 1521

[8] Y. Lu, J. Liu, Acc. Chem. Res. 2007, 40, 315.

[9] K. Lund, A. J. Manzo, N. Dabby, N. Michelotti, A. Johnson-Buck, J. Nangreave, S. Taylor, R. Pei, M. N. Stojanovic, N. G. Walter, E. Winfree, H. Yan, Nature 2010, 465, 206.

[10] Y. Chen, M. Wang, C. Mao, Angew. Chem., Int. Ed. 2004, 43, 3554.

[11] G. Bonner, A. M. Klibanov, Biotechnol.Bioeng. 2000, $68,339$.

[12] T. Herskovits, S. J. Singer, Arch. Biochem. Biophys. 1961, 94, 99.

[13] A. F. Usatyi, L. S. Shlyakhtenko, Biopolymers 1974, 13, 2435.

[14] J. Piskur, A. Rupprecht, Febs Letters 1995, 375, 174.

[15] S.-i. Nakano, D. Miyoshi, N. Sugimoto, Chem. Rev. 2014, 114, 2733.

[16] A. Beg Menhaj, B. Smith, J. Liu, Chem. Sci. 2012, 3, 3216.

[17] B. D. Smith, J. Liu, J. Am. Chem. Soc. 2010, 132, 6300.

[18] D. B. Knowles, A. S. LaCroix, N. F. Deines, I. Shkel, M. T. Record, Proc. Natl. Acad. Sci. U.S.A. 2011, 108, 12699.

[19] H. Sigel, R. Tribolet, J. Inorg. Biochem. 1990, 40, 163.

[20] A. L. Feig, G. E. Ammons, O. C. Uhlenbeck, RNA 1998, 4, 1251.

[21] R. K. O. Sigel, A. M. Pyle, Chem. Rev. 2007, 107, 97.

[22] S.-i. Nakano, Y. Kitagawa, H. Yamashita, D. Miyoshi, N. Sugimoto, ChemBioChem 2015, 16, 1803.

[23] A. K. Behera, K. J. Schlund, A. J. Mason, K. O. Alila, M. Han, R. L. Grout, D. A. Baum, Biopolymers 2013, 99, 382.

[24] Y. Lu, Chem. Eur. J. 2002, 8, 4588.

[25] W. L. Ward, K. Plakos, V. J. DeRose, Chem. Rev. 2014, 114, 4318.

[26] P.-J. J. Huang, J. Lin, J. Cao, M. Vazin, J. Liu, Anal. Chem. 2014, 86, 1816. 
[27] P.-J. J. Huang, M. Vazin, J. Liu, Anal. Chem. 2014, 86, 9993.

[28] P.-J. J. Huang, M. Vazin, Ż. Matuszek, J. Liu, Nucleic Acids Res. 2015, 43, 461.

[29] P.-J. J. Huang, J. Liu, Nucleic Acids Res. 2015, 43, 6125.

[30] Y. Li, D. Sen, Nat. Struct. Biol. 1996, 3, 743.

[31] P. Travascio, Y. Li, D. Sen, Chem. Biol. 1998, 5, 505.

[32] L. Stefan, F. Denat, D. Monchaud, Nucleic Acids Res. 2012, 40, 8759.

[33] R. R. Breaker, G. F. Joyce, Chem. Biol. 1994, 1, 223.

[34] Y. Li, R. R. Breaker, J. Am. Chem. Soc. 1999, 121, 5364.

[35] S.-F. Torabi, P. Wu, C. E. McGhee, L. Chen, K. Hwang, N. Zheng, J. Cheng, Y. Lu, Proc. Natl. Acad. Sci. U.S.A. 2015, 112, 5903.

[36] A. Roth, R. R. Breaker, Proc. Natl. Acad. Sci. U.S.A. 1998, 95, 6027.

[37] V. K. Jayasena, L. Gold, Proc. Natl. Acad. Sci. U.S.A. 1997, 94, 10612.
[38] D. Mazumdar, N. Nagraj, H.-K. Kim, X. Meng, A. K. Brown, Q. Sun, W. Li, Y. Lu, J. Am. Chem. Soc. 2009, 131, 5506

[39] J. B. Murray, A. A. Seyhan, N. G. Walter, J. M. Burke, W. G. Scott, Chem. Biol. 1998, 5, 587.

[40] C. R. Geyer, D. Sen, Chem. Biol. 1997, 4, 579.

[41] A. A. Seyhan, J. M. Burke, RNA 2000, 6, 189.

[42] A. Terol, E. Paredes, S. E. Maestre, S. Prats, J. L. Todolí, Journal of Chromatography A 2011, 1218, 3439.

[43] H. Li, X.-S. Chai, Y. Deng, H. Zhan, S. Fu, Journal of Chromatography A 2009, 1216, 169.

[44] L. Zhang, H. Qi, Y. Wang, L. Yang, P. Yu, L. Mao, Anal. Chem. 2014, 86, 7280.

[45] M. Akamatsu, T. Mori, K. Okamoto, H. Komatsu, K. Kumagai, S. Shiratori, M. Yamamura, T. Nabeshima, H. Sakai, M. Abe, J. P. Hill, K. Ariga, ACS Appl. Mater. Inter. 2015, 7, 6189.

[46] S. Petrova, Y. Kostov, K. Jeffris, G. Rao, Anal. Lett. 2007, 40, 715. 


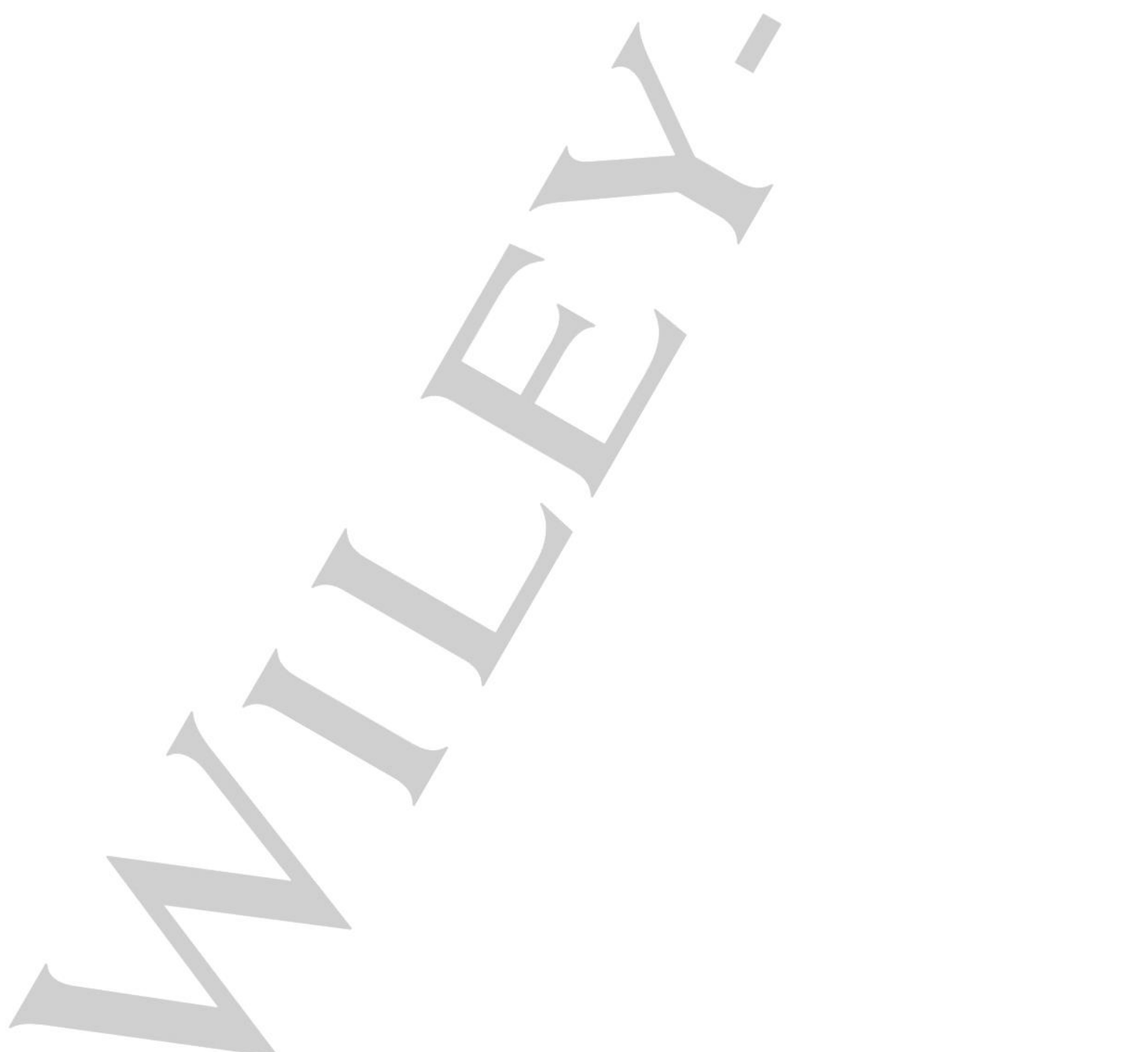

